\title{
Phase Synchronization of a Two-Channel Phase-Sensitive Amplifier based on Optical Injection-Locking of InP Quantum-Dash Mode-Locked Lasers
}

\author{
Ehsan Sooudi $^{1,2}$, Stylianos Sygletos ${ }^{2,3}$, Paola Frascella ${ }^{2,3}$, Andrew D. Ellis ${ }^{2,3}$, Guillaume Huyet ${ }^{1,4}$, John G. \\ McInerney $^{1,2}$, Francois Lelarge ${ }^{5}$, Kamel Merghem ${ }^{6}$, Ricardo Rosales ${ }^{6}$, Anthony Martinez ${ }^{6}$, Abderrahim \\ Ramdane $^{6}$, and Stephen P. Hegarty ${ }^{1,4}$ \\ ${ }^{1}$ Optoelectronics Group, Tyndall National Institute, Lee Maltings, Cork, Ireland \\ ${ }^{2}$ Department of Physics, University College Cork, Cork, Ireland \\ ${ }^{3}$ Photonic Systems Group, Tyndall National Institute, Lee Maltings, Cork, Ireland \\ ${ }^{4}$ Centre for Advanced Photonics and Process Analysis (CAPPA), Cork Institute of Technology, Cork, Ireland \\ ${ }^{5}$ Alcatel-Thales III-V Lab, a joint Laboratory of "Alcatel Lucent Bell Labs", "Thales Research and Technology", and "CEA-LETI",91460, \\ Marcoussis, France \\ ${ }^{6}$ Laboratory for Photonics and Nanostructures, CNRS, Marcoussis 91460, France \\ Email: (Ehsan.Sooudi@,Tyndall.ie)
}

\begin{abstract}
A synchronization scheme for a two-channel phase sensitive amplifier is implemented based on the injection-locking of single InP quantum-dash mode-locked laser. Error free performance with penalty $<1 \mathrm{~dB}$ is demonstrated for both channels.

(C)2011 Optical Society of America

OCIS codes: (190.4380) Nonlinear optics, Four-wave mixing, (060.1155) All-optical networks, (140.3520) Lasers, injection-locked, and (140.4050) Mode-locked lasers.
\end{abstract}

\section{Introduction}

Phase sensitive amplifiers (PSAs) have recently attracted a lot of interest due to their unique phase squeezing capabilities, which makes them an ideal technology platform for regenerating phase encoded modulation formats, i.e. differential phase shift keying (DPSK) [1]. Although their phase squeezing capabilities have been well known in literature for more than a decade [2], a practical implementation that would allow them stand as independent, "black-box" elements in transmission links was missing until recently [3]. This was achieved using the synchronization scheme proposed in [4], which combined carrier extraction and frequency generation functionalities along with optical injection-locking of a local laser to create the synchronized pumps of the PSA. Recently, this scheme was extended to demonstrate the first two-channel DPSK PSA using optical injection-locking of two independent single-mode lasers [5].

However, increasing the number of channels in such a multi-pump "black-box" PSA, requires at its synchronization stage an equal number of additional local pumps, each one of them phase-locked to the corresponding extracted carrier. This makes the overall setup complicated and bulky; therefore, a solution that would enable multiple pump generation by synchronizing a single laser comb-source is expected to be preferable. The possibility of coherent frequency comb generation using dual-mode (two coherent lines) injection locking of InP quantum-dash mode-locked lasers (QDMLLs) has recently been demonstrated [6]. This capability enabled phase synchronization of many comb lines to only two coherent $\mathrm{CW}$ lines. In this paper, we demonstrate a powerful application of this injection-locking technique, which allowed us to create multiple synchronized pumps for phase sensitive amplification using a single QDMLL which was injection-locked by two extracted signal carriers. With the proposed scheme, phase sensitive performance was achieved with a gain swing larger than $7 \mathrm{~dB}$ as well as error free operation with a penalty of less than $1 \mathrm{~dB}$ for two optically phase-locked WDM channels.

\section{Experimental arrangement and results}

The experimental set up is illustrated in Fig. 1. At the transmitter a comb generator consisting of two cascaded Mach-Zehnder modulators was used to create synchronized optical carriers spaced at $41.496 \mathrm{GHz}$. Two of them were selected at $\lambda_{s l}=1558.431 \mathrm{~nm}$ and $\lambda_{s 2}=1558.759 \mathrm{~nm}$ and modulated in DPSK format at $10.374 \mathrm{Gbit} / \mathrm{s}$ with a pseudorandom binary sequence (PRBS) of $2^{7}-1$. Subsequently, the channels were decorelated by $20 \mathrm{~km}$ of conventional single mode fiber (SMF) and amplified by an EDFA at $11 \mathrm{dBm}$. At the carrier extraction stage of the PSA, two input signals mixed with a local DFB laser emitting at $\lambda_{p 1}=1554.898 \mathrm{~nm}$ and were amplified to $30 \mathrm{dBm}$ in a highly nonlinear fiber (HNLF). The HNLF had a length of $0.198 \mathrm{~km}$, dispersion parameter $-0.2 \mathrm{ps} /(\mathrm{nm} \cdot \mathrm{km})($ at $1550 \mathrm{~nm}$ ), nonlinear coefficient $7.4 \mathrm{~W}^{-1 \cdot} \mathrm{km}^{-1}$ and attenuation of $14 \mathrm{~dB} / \mathrm{km}$ [7]. At the HNLF output the two generated carriers were self-locked to the corresponding signals and the local pump and located at symmetric wavelengths 
with respect to them, as shown in Fig. 2(a). The output of the HNLF was divided in two arms. In one arm, we used a band pass filter ( $3 \mathrm{~dB}$ bandwidth $4 \mathrm{~nm})$ and two interleavers $(50 \mathrm{GHz}$ and $21.31 \mathrm{GHz}$, respectively) to select the carriers and clean them from the residual frequencies of the FWM process. Then the filtered spectrum, shown in Fig. 2(b), was injected through a circulator to the QDMLL. The circulator was connected to the QDMLL through a lensed fibre introducing a typical loss of $3 \mathrm{~dB}$. The output of the laser $(-1.2 \mathrm{dBm})$ was then amplified by a $13 \mathrm{dBm}$ EDFA and combined with the pump and signal spectrum of the other arm to a Wavelength Selective Switch (WSS). The WSS also removed unwanted frequencies from the carrier extraction process and selected the two signal channels and the three pumps; see Fig. 2(c). The output of WSS was amplified by a $30 \mathrm{dBm}$ high power EDFA before launched to a second HNLF (with the same parameters as first HNLF and with a length of $0.19 \mathrm{~km}$ ) to form the PSA. We used a conventional feedback based phase-locked loop circuit to control a piezoelectric-based fibre stretcher (PZT) and compensate any slow thermal or acoustic induced phase drift between the interacting waves in both paths.

The QDMLL used in this experiment was a two-section device with total cavity length of $2030 \mu \mathrm{m}$, with $50 \mu \mathrm{m}$ $(\approx 2.5 \%)$ saturable-absorber section operating as self-mode-locked lasers [8] when the absorber was left floating, giving mode-locked frequency around $20.8 \mathrm{GHz}$. The device was mounted p-side up on an AlN submount and copper block with active temperature control.

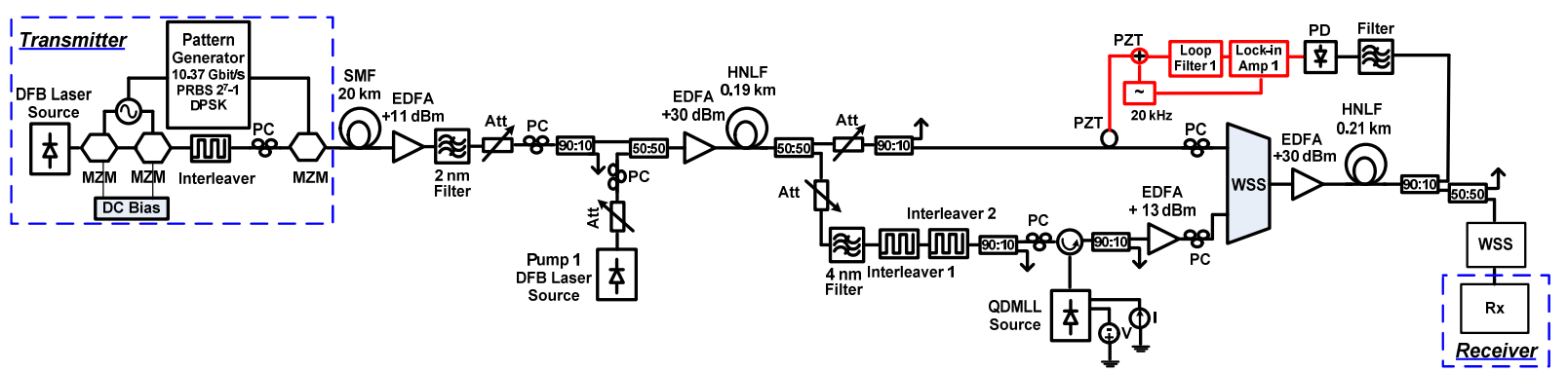

Fig. 1. Experimental setup for two-channel DPSK phase sensitive amplifier based on QDMLL phase synchronization.

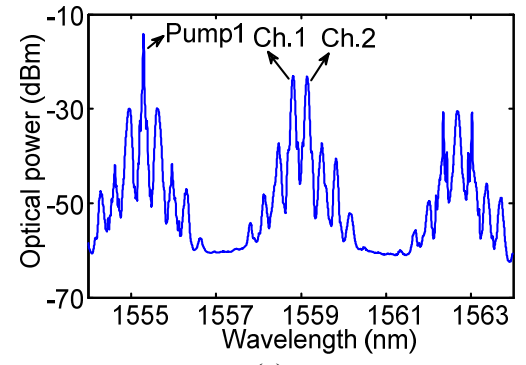

(a)

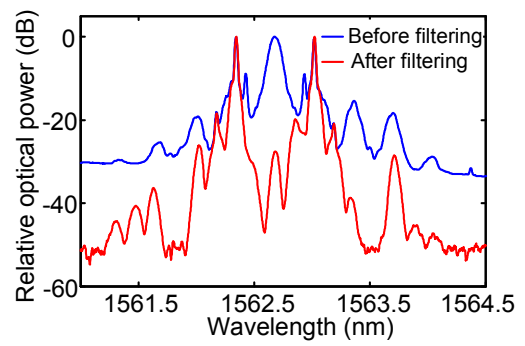

(b)

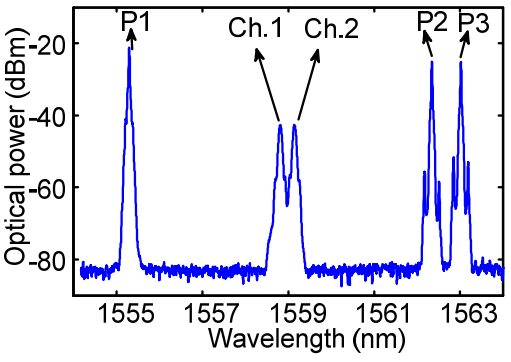

(c)

Fig. 2. (a). Optical spectrum of first HNLF output. (b). Optical spectrum of extracted carriers before filtering (blue-solid line) and after filtering stages (red-solid line). (c). Optical spectrum of PSA input, after removing un-wanted components using WSS.

The optical spectrum of the injected signal and injection-locked QDMLL is shown in Fig. 3(a). As can be seen, the injected modes had higher power than the non-injected ones by $>5 \mathrm{~dB}$ indicating they had more share of the total power of the injection-locked comb. This is beneficial for this scheme, as only the injected modes were used as pump sources. Despite the fact that the spacing of extracted carriers were four times the repetition rate of injectionlocked laser, the same locking mechanism as dual-mode injection at first harmonic occurred [6]. The RF spectrum of the laser when free running (without injection) and under injection-locking is depicted in Fig. 3(b), where significant RF linewidth narrowing was observed. The Lorentzian fit to the RF linewidth of the free running laser was $270 \mathrm{kHz}$ (not shown) whereas the RF linewidth of the injection-locked laser reduces to values beyond the resolution bandwidth (RBW) of the instrument. The inset in Fig. 3(b) shows the $80 \mathrm{~Hz}$ zoom of the peak with resolution bandwidth of $1 \mathrm{~Hz}$; the $20 \mathrm{~dB}$ linewidth of the tone is estimated to be $3 \mathrm{~Hz}$, indicating strong reduction of timing jitter of the device. This confirms the presence of phase-locking between the injected modes and extracted carriers. In comparison with dual-mode injection, we observed less RF signal to noise ratio in the wide RF span which is due to the additional noise generated as a result of imperfect carrier extraction scheme.

In order to suppress the residual phase modulation present in the extracted carriers, the injection ratio (ratio of injected power to the free running slave's power) should be kept as low as possible [9] (about $-30 \mathrm{~dB}$ in this 
experiment). The phase sensitive amplifier was stabilized against thermal fiber expansion using a conventional phase-locked loop [5] and was observed at maximum and minimum gain for both channels, as shown in Fig. 3(c). A $7.4 \mathrm{~dB}$ on-off gain was measured for both channels which may be increased by using higher pump powers.

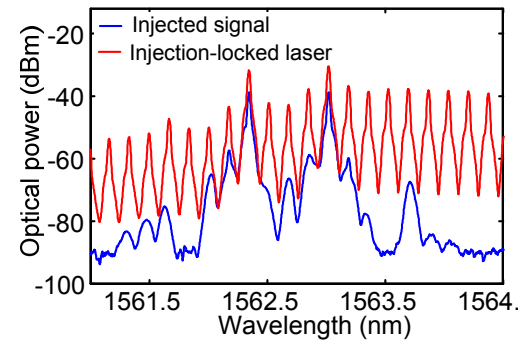

(a)

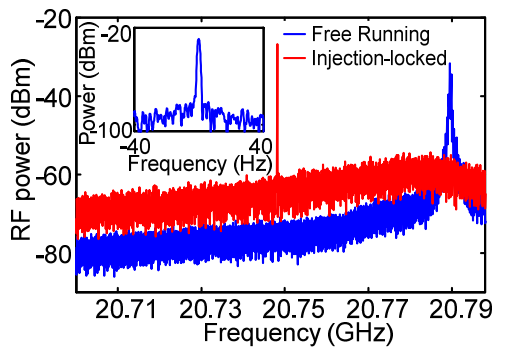

(b)

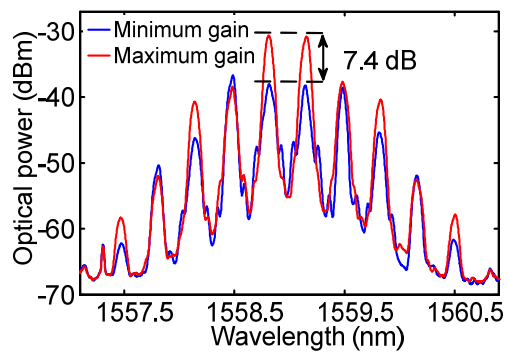

(c)

Fig. 3. (a). Optical spectrum of injected signal (blue-solid line) and injection-locked QDMLL (red-solid line). (b). RF spectrum of free running (blue-solid line) and injection-locked QDMLL (red-solid line), the inset shows a zoom of the RF tone with $80 \mathrm{~Hz}$ span and RBW of 1 Hz. (c). The zoom of the optical spectrum at the PSA output around channels for maximum (red-solid line) and minimum (blue-solid line) gain.

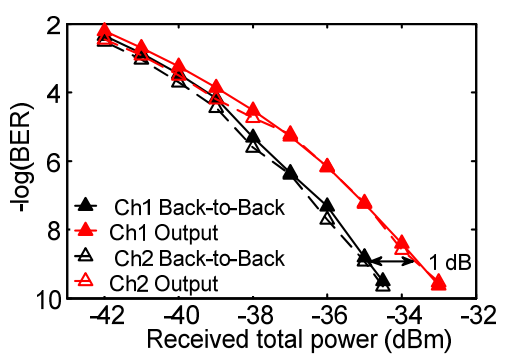

(a)

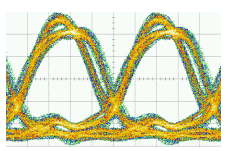

Ch. 1 Back-to-Back

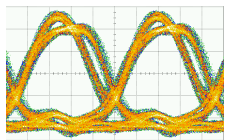

Ch. 2 Back-to-Back

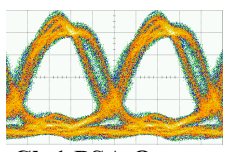

Ch.1 PSA Output

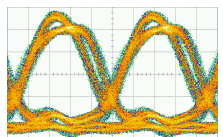

Ch. 2 PSA Output

(b)

Fig. 4. (a). BER measurements of both channels versus total received power. (b). Error free eye diagrams for both channels at the input and output of the PSA.

We also studied the performance of PSA in terms of BER measurements for both channels. Fig. 4(a) depicts the BER for both channels in back-to-back and PSA output (maximum gain) against received power. By comparing the BER curves for both channels at the output, about $1 \mathrm{~dB}$ sensitivity penalty for BER of $10^{-9}$ was observed, with negligible penalty at the FEC threshold around $10^{-3}$. The eye diagrams for both channels at the output are open proving the excellent performance of our scheme; see Fig 4(b).

\section{Conclusion}

We demonstrated a two-channel 10.374 Gbit/s DPSK "black-box" PSA based on simultaneous phase synchronization of local pumps to incoming signals through novel injection-locking technique using a $21 \mathrm{GHz} \mathrm{InP}$ QDMLL. 7.4 dB phase sensitive gain and only $1 \mathrm{~dB}$ sensitivity penalty observed in the BER measurements of two channels at the output of the amplification stage. This scheme has the potential for extension to multiple channels towards more compact and efficient "black-box" PSAs.

This work has been supported by the Science Foundation Ireland (SFI) under Contract 07/IN.1/I929 and also by European Communities Seventh Framework Programme FP/2007-2013 under grant agreement 224547 (PHASORS).

\section{References}

[1] K. Croussore and G. Li, "Phase and amplitude regeneration of differential phase-shift keyed signals using phase-sensitive amplification," IEEE J. Sel. Topics Quantum Electron.. vol. 14, no. 3, pp. 648-658, 2008.

[2] M. E. Marhic, et.al, "Optical amplification in a nonlinear fibre interferometer," Electron. Lett., vol.27, no.3, pp 210-211, (1991).

[3] R. Slavík, et al., "All-optical phase and amplitude regenerator for next-generation telecommunications systems,” Nat. Photon., 4, 690-695 (2010).

[4] R. Weerasuriya et al. "Generation of frequency symmetric signals from a BPSK input for phase sensitive amplification,” in Proc. OFC 2010, paper OWT6.

[5] P. Frascella, et al., "Two channel phase regeneration based on a "black-box" phase sensitive amplifier," In Proc. ECOC 2011.

[6] E. Sooudi, et al., "Injection locking properties of InAs/InP-based mode-locked quantum-dash lasers at 21 GHz," IEEE Photon. Technol. Lett., vol. 23, no. 20, pp. 1544-1546, 2011.

[7] L. Gruner-Nielsen, et al., "A silica based highly nonlinear fibre with improved threshold for stimulated brillouin scattering," in Proc. ECOC 2010, paper Tu.4.D.3.

[8] F. Lelarge et al., "Recent advances on InAs/InP quantum dash based semiconductor lasers and optical amplifiers operating at 1.55 m," IEEE J. Sel. Topics Quantum Electron., vol. 13, no. 1, pp. 111-124, Jan./Feb. 2007.

[9] S Sygletos et al., "Phase synchronization scheme for a practical phase sensitive amplifier of ASK-NRZ signals," Opt. Exp., vol. 19, no. 13, pp. 12384-12391. 